www.nature.com/ejhg

\title{
Author index to volume 10
}

Key:

(BR) Book review; (C) Corrigendum; (CP) Course profile; (CSR) Case report; (E) Editorial; (ER) Erratum; (GP) Genetic policy; (L) Letter; (MR) Meeting report; (PG) Practical genetics; (R) Review; (SR) Short report; (VP) Viewpoint

\author{
Aasly JO 773 \\ Abbott A 757 \\ Abecasis G 266 \\ Abel J 499 \\ Abkevich V 539 \\ Adato A 339 \\ Adolfsson R 276 \\ Afman LA 433 \\ Akar S $786(\mathrm{~L})$ \\ Akkoç N $786(\mathrm{~L})$ \\ Akyerli C 77 (SR) \\ Al-Taie OH 499 \\ Ala-Kokko L 562 \\ Albrecht B 790 \\ Albrecht M 729 \\ Aldashev AA 741 \\ Aldred MJ 865 \\ Alitalo T 449 \\ Allamand V 91 (PG) \\ Alliende MA 638 \\ Allikmets R 197 \\ Alonso A 553 \\ Alves C 467 \\ Amiel J 699 \\ Andersen PM 271 \\ Anderson GG 266 \\ Anderson LVB 825 \\ Andersson K 52 \\ Antoniadi T 694 \\ Antonicelli R 292 \\ Antonucci I 388 (SR) \\ Appel S 17 \\ Arieff Z 36 \\ Armengol L 26 \\ Arndt S 17 \\ Arnett DK 367 \\ Arpa J 773 \\ Arvanitis DA 113 \\ Asherson P 857 \\ Aubé J 150 (L) \\ Audrézet M-P 100 \\ Avidan N 339
}

\begin{tabular}{|c|c|}
\hline Avraham KB 339 & Bittner R 427 \\
\hline \multirow[t]{2}{*}{ Azoulay D 239} & Blackwood D 276 \\
\hline & Blanchet P 72 \\
\hline Bader JS 125, 870 & Blennow K 113, 579 \\
\hline Baker P 491 (SR) & Blin N 95 \\
\hline Bakou K 694 & Blom HJ 433 \\
\hline Balcells S 197 & Bocciardi R 183 \\
\hline Baldwin CT 883 (SR) & Bohlega S 773 \\
\hline Ballabio A 813 & Boileau C 673 \\
\hline Ballinger DG 539 & Boily G 62 \\
\hline Bao X-H 484 (SR) & Bonafè M 292 \\
\hline Barash V 226 & Bonardi G 183 \\
\hline Barbieri M 292 & Bonaventure J 819 \\
\hline Bardien-Kruger S 36 & Bonduelle M 591 \\
\hline Bardtke B 95 & Bonk T 188 \\
\hline Barone N 362 & Bonne G 157 (PG) \\
\hline Barros A 467 & Bonne-Tamir B 339 \\
\hline Baumgartner W-D 427 & Boocock G 250 \\
\hline Becker C-M 188 & Boon LM 375 \\
\hline Becker K 188 & Booth DR $786(\mathrm{~L})$ \\
\hline Beckmann JS 339 & Booth SE $786(\mathrm{~L})$ \\
\hline Behrend C 790 & Borecki I 367 \\
\hline Belenkiy O 339 & Borghini S 183 \\
\hline Belliard S 399 & Børglum AD 276 \\
\hline Belohradsky BH 217 (SR) & Børresen-Dale A-L 521 \\
\hline Ben-Asher E 339 & Bouccara D 851 \\
\hline Benitez J 457 & Bouchard J-P 406 \\
\hline Berardelli M 292 & Bourgain C 313 \\
\hline Berg J 807 & Boyer A 297 \\
\hline Bergbaum A 807 & Boyle S 833 \\
\hline Bergheim A 17 & Bragliani M 137 \\
\hline Bernard R 297 & Brand E 715 \\
\hline Bernardi F 576 & Breedveld G 162 \\
\hline Bertorelle G 530 & Bressac-de Paillerets B 599 \\
\hline Bhattacharya SS 197, 245 & Brice A 399 \\
\hline Bhattacharyya S 177, 266 & Briggs PJ 749 \\
\hline Bibi CN 226 & Brik R 145 \\
\hline Binzer M 271 & Brin $M 773$ \\
\hline Bird AC 197, 245 & Brink P 36 \\
\hline Birkner T 334 (SR) & Broghammer M 449 \\
\hline Bisgaard ML 631, 887 (E) & Brookes AJ 153 (MR) \\
\hline Bitoun P 516 & Brown CJ 44 \\
\hline
\end{tabular}

\author{
Broxholme J 266 \\ Brun M-E 833 \\ Brunner HG 197, 487 (SR) \\ Bubel S 204 \\ Bulow S 631, 887 (E) \\ Bürk K 204 \\ Burn J 516 \\ Burrello N 362 \\ Bygren LO 682
}

Caballero IR 773

Caillaud C 511

Calabrese G 388 (SR)

Callens T 334 (SR)

Calogero AE 362

Campion D 399

Camuzat A 399

Cantor RM 547

Cardelli M 292

Cardim N 741

Cardon LR 616

Carmi N 724

Carvalho F 467

Cascon A 457

Cassiman J-J 505

Castoldi E 576

Cavé-Riant F 733

Cavalleri GL 521

Cavalli-Sforza L-L 521

Cavallone L 292

Cebrian A 457

Ceccherini I 183

Cécillon M 733

Chai J 162

Chalmers RM 773

Chandy KG 36

Chapiro E 851

Chapman K 562

Chardenoux S 210

Chatelain N 715

Chemaly MM 841

Chen J-M 100 
Cherny SS 125

Chioza B 857

Chiurazzi P 767

Chouery E 210, 391 (SR)

Chouinard S 773

Christophers E 327

Christopoulou S 694

Cimbis M 77 (SR)

Cisternino M 137

Clark K 150 (L)

Claussen U 790

Claustres M 72

Clerget-Darpoux F 313, 399

Collod-Béroud G 673

Conconi F 576

Cookson WOCM 177, 266

Coon H 367, 539

Cooper C 749

Coppola A 579

Corfield V 36

Cormier-Daire V 699

Correia JM 741

Cortés F 638

Couderc R 851

Cox R 266

Cremades N 467

Cremers FPM 197

Croke DT 530

Croquette M-F 107

Cuccia M 137

Culross F 583

Curran ME 259

Curtis D 491 (SR)

Dagan E 724

D'Agata R 362

Dallapiccola B 303

Dalski A 204

Danek A 773

D'Angelo A 579

Davies A 807

Davis LM 707

Day INM 749

De Backer G 841

De Bacquer D 841

De Benedictis G 119, 292

De Blois M-C 699

Debuire B 239

De Cássia M Pavanello $\mathrm{R}$ 825

Degn B 381

De Grève J 879 (SR)

De La Chapelle A 449

Delague V 391 (SR)

Del-Favero J 276
Delobel B 107

Demenais F 715

Dengler R 773

Denier C 733

Dennis NR 707

Dennison EM 749

Denoyelle F 851

De Palma A 362

De Paula F 825

Depetris D 107

De Rienzo A 95

De Rijcke M 591

De Sario A 833

Dessay B 516

DeStefano AL 883 (SR)

De Vos A 591

Devoto M 95

De Vries A 819

De Vries BBA 487 (SR)

Devroey P 591

Didic M 399

Dietz R 741

Diez JJ 457

Do Carmo Martins M 841

Dobson-Stone C 773

Doll A 351

Domènech E 421

Dondi E 137

Donnelly JG 578

Donoghue J 694

Doran RM 245

Dotti MT 773

Dourisboure RJ 395 (SR)

Dowling B 562

Downey LM 865

Drugan C 511

Duba H-C 351

Dubé M-P 406

Dubois B 399

Ducrocq R 320

Dumanski JP 52

Dupré N 773

Durie PR 250

Dürr A 167, 689

D'Urso M 197

Dussau J 511

Eckart M 334 (SR)

Eckfeldt JH 367

Edvinsson S 682

Eerola I 375

Ehnholm C 841

Ehtesham N 250

Eisinger F 599

Elder JT 327
Eliot M-M 851

Ellis L 250

Ellison RC 367

Emile J-F 239

Erdel M 351

Ermert A 753

Espinos C 339

Estivill X 26

Evans A 841

Evers-Kiebooms G 167, 689

Ewald H 276, 381

Faivre L 699

Fall CHD 749

Fan R 607

Fananapazir L 773

Farrall M 553

Farrell SA 44

Fayle S 865

Federico A 773

Felder B 753

Feldmann D 851

Ferec C 100

Ferlin A 303

Ferrante M-I 516

Ferrante P 813

Filter M 17

Finch A 150 (L)

Fisher SA 259

FitzPatrick D 807

Flannery JG 339

Flothmann K 197, 883 (SR)

Fonatsch C 334 (SR)

Fontes M 223 (PG)

Forbes A 259

Foresta C 303

Forrest SM 623

Forsgren L 271

Franceschi C 119, 292

Franco B 516

Fransen E 883 (SR)

Frei $\mathrm{K} 427$

Friedman E 724

Fryer AE 773

Fryns J-P 505

Garabédian E-N 851

Gardes N 231

Gaspari AR 388 (SR)

Gates GA 883 (SR)

Gatta V 388 (SR)

Gaunt TR 749

Gécz J 767

Geller F 753

Genin E 313
Geraud G 773

Gershoni-Baruch R 145, 724

Geuzens E 643

Gianaroli L 303

Giedraitis V 271

Giordano PC 672

Giovagnetti S 292

Giurgea I 699

Goelen G 879 (SR)

Goldfarb LG 773

Golfier V 399

Golla A 217 (SR)

Gómez-Zaera M 421

Gonzàlez-Duarte R 197

Goobie S 250

Gosset P 699

Gratacòs M 26

Grazioso C 362

Green MW 583

Greener MJ 413

Grigorescu-Sido P 511

Grigoriadou M 694

Grynberg H 375

Grzeschik K-H 351

Gueresi P 292

Guicheney P 91 (PG)

Guitart M 26

Guo S-W 327

Gurling HM 381, 491 (SR)

Gutin A 539

Gyftodimou J 694

Haahtela T 658

Haddy N 841

Hall JM 82 (SR)

Halley D 783

Hamalainen R 339

Hamel BCJ 487 (SR)

Hammerer I 351

Hampe J 259

Hanauer A 2 (PG)

Hannequin D 399

Hansmann I 790

Hardie RJ 773

Harper P 167, 689

Hartsfield JK 516

Hatzipouliou A 694

Hawkins PN 786 (L)

He B 271

Heinonen S 569

Helbling-Leclerc A 157 (PG)

Helisalmi S 569

Hellebrand H 217 (SR)

Hellenbroich Y 204

Heller A 790 
Hemmrich N 197

Henn W 790

Hennies HC 17

Henseler T 327

Hérasse M 666

Hergersberg M 449

Heutink P 162

Hide W 17

Hiersemenzel L-P 773

Hillert J 271

Hiltunen M 569

Hims MM 245

Hjermind LE 213 (SR)

Holmberg J 475

Holmdahl R 475

Hopkins PJ 82 (SR)

Hopkins PN 367, 539

Howard F 707

Howard HC 406

Hoyng CB 197

Hsu H-M 495 (SR)

Huang S 162

Hugel L 150 (L)

Humeny A 188

Humpries SE 782

Hunt SC 367, 539

Hutchinson M 141

Ikeda Y 773

Imreh S 52

Ingelhearn CF 245, 865

Ingvast S 197

Isotalo PA 578

Ivezič S 276

Jack E 150 (L) Jacopini G 167, 689

Jacquot $S 2$ (PG)

Jafara-Boroujerdi M 188

Jakob F 499

Jalili IK 865

Jansson A 217 (SR)

Jardel C 851

Jaunsolo MA 457

Jawaid A 125

Jebeleanu G 511

Jenisch S 327

Joensuu T 339

Johnson S 773

Jonveaux P 462

Jorge P 197

Jorgensen TH 381

Joutel A 733

Joyce CA 707

Jue $Y 162$
Julian-Reynier C 599

Kaati G 682

Kabaeva ZT 741

Kaitila I 439

Kalsi G 381, 491 (SR)

Kaplan J 197, 516

Karatzis P 694

Karp BI 773

Katsarou E 694

Kauppinen R 649

Keeling J 807

Keen TJ 245, 865

Kelbova C 790

Kere J 658

Kirk KM 616

Kirschhofer K 427

Kiss C 52

Kiss $\mathrm{H} 52$

Kleefstra T 487 (SR)

Klein G 52

Knight J 857

Koch MC 753

Koçoglu SR 77 (SR)

Köhrle J 499

König K 95

Kovanen PT 547

Kpebe A 86 (SR)

Krajc M 879 (SR)

Krishnamoorthy R 320

Kronenberg F 367, 539

Kruse TA 276, 381

Kukuruga D 327

Kung C-Y 495 (SR)

Kwan E 150 (L)

Laakso M 547

Laasanen J 569

Labauge P 733

Laberge-le Couteulx S 733

Lacomblez L 399

Lahdenperä S 547

Laitinen LA 658

Laitinen T 658

Lamb G 491 (SR)

Lancet D 339

Lange EM 327

Larizza D 137

Lathrop GM 553

Lathrop M 210, 391 (SR), 715

Latour P 297

Le Maréchal C 100

Leaves NI 177, 266

Lees A 773
Lefort G 462

Lehesjoki A-E 339

Lemainque A 210

Lemoine A 239

Lennard-Jones JE 259

Leqius E 505

Leroy JG 334 (SR)

Leshinsky-Silver E 226

Lev D 226

Levy E 266

Lévy N 297

Lewis CM 259

Li H 162

Li M 162

Li S-Y 495 (SR)

Liebaers I 591

Liede A 150 (L)

Liehr T 790

Lievers KJA 433

Ligers A 271

Lin AA 521

Lin C-C 495 (SR)

Lisa R 292

Lissens W 591

Livneh A 145

Lo WHY 162

Loiselet J 210

Loiselet J 391 (SR)

Lopez-lbarra PJ 457

Lorenz B 449

Lossos A 773

Loughlin J 562

Lovett M 17

Lu S 475

Lucas T 427

Lurkin I 819

Maciazek J 733

Mackey DA 245

Macpherson AJS 259

Maddison P 773

Mäkitie O 439

Makoff AJ 857

Male A 807

Malzac P 297

Mancini J 86 (SR)

Mann C 351

Mannermaa A 569

Mansfield DC 245

Mantovani V 137

Maotti J-P 599

Marcelline L 562

Marchegiani F 292

Markham AF 245

Marlin S 851
Marquardt A 281 (SR)

Marra M 292

Martens K 643

Martin S 553

Martinetti M 137

Matera I 183

Mathew CG 259

Mathieu J 406

Matioli SR 825

Matsuda F 553

Mattei M-G 107

Matthijs G 505, 643, 783

Maugeri A 197

Maurice M 841

Mayet S 491 (SR)

Mayne PD 530

Mazzoni G 576

McCormick J 583

McDonald HL 44

McHale J 865

McKie AB 245

McQuillin A 381, 491 (SR)

Medlej-Hashim M 391 (SR)

Mégarbané A 210, 391 (SR)

Mehta A 583

Mehta G 583

Meindl A 217 (SR), 449

Meiner V 773

Meitinger T 217 (SR)

Mendlewicz J 276

Meng HD 484 (SR)

Mentrup B 499

Mercado FC 773

Meroni G 813

Messali S 813

Messiaen L 334 (SR)

Metspalu A 197

Michils G 505

Mighell A 865

Mignon-Ravix C 107

Millán JM 339

Mirghomizadeh F 95

Mirrakhimov MM 741

Missirian C 86 (SR)

Mohiddin S 773

Monaco AP 773

Moncla A 86 (SR)

Mondain M 72

Montpetit A 62

Moore AT 245

Moorey H 491 (SR)

Moraine C 516

Moreaud O 399 
Morizio E 388 (SR)

Mörk H 499

Mornet E 666

Morris AAM 141

Morrison J 250

Mors O 276, 381

Moutou C 231

Mueller RF 245

Mühlbauer M 334 (SR)

Muir W 276

Mulliken JB 375

Munnich A 289 (VP), 699

Muntoni F 413

Mustafa Z 562

Mustajoki P 649

Mustapha M 210, 391

(SR)

Myers RH 883 (SR)

Naboulsi M 210

Nadal M 26

Nair R 327

Nanda I 281 (SR)

Narod SA 150 (L)

Nashef L 857

Nassogne M-C 699

Naughten E 530

Nedelcu R 150 (L)

Nègre $P 297$

Nemeth A 516, 773

Neri G 767

Newbury-Ecob R 516

Neyer M 351

Nielsen FC 631, 887 (E)

Nigro V 825

Nillesen W 487 (SR)

Nordquist N 475

Nunes V 421

Nuotio 10547

Nys K 167, 689

O'Brien J 757

O'Connell JR 133

O'Connor RD 82 (SR)

Odent S 699

O'Dell SD 749

O'Donnell KA 530

Oeken I 95

Ogilvie CM 801, 807

Ogilvie E 17

Öktem S 786 (L)

Olender T 339

Olivieri F 292

Olofsson P 475

Oostra BA 162
O'Neill C 530

Oruč L 276

Osei-Lah A 857

Osterziel KJ 741

Oudakker AR 487 (SR)

Oyen N 516

Ozçelik T 77 (SR)

Pafumi C 362

Paillard F 715

Pajukanta P 537

Palka G 388 (SR)

Pallares-Ruiz N 72

Palmer LJ 82 (SR)

Palmer M 113

Palmqvist L 113

Paloma E 197

Pan H 484 (SR)

Paolisso G 292

Paperna T 724

Parronaud J 210, 391 (SR)

Pasquier L 699

Passarino G 521

Passeri G 292

Passos-Bueno MR 825

Patel R 197

Peltonen L 547

Pembrey ME 669

Perrot A 741

Petäys T 658

Petersen MB 694

Petit C 210, 391 (SR), 851

Pettersson U 475

Pfister M 95

Philip N 297

Philippe C 462

Phillips DIW 749

Pieri C 292

Pietrokovski S 339

Pihlajamäki J 547

Plass C 6 (R)

Platteau P 591

Podesta EJ 395 (SR)

Poenaru L 511

Polvi A 658

Polvi T 658

Popovic M 250

Prévost C 406

Prieur M 699

Procopciuc L 5112

Province MA 367

Puel M 399

Pujana MA 26

Punnonen K 569

Purcell S 125
Pusch CM 449

Quéré I 100

Quested D 491 (SR)

Raap AK 427

Radvanyi F 819

Raguénès O 100

Rahil H 462

Räinä SS 562

Rampoldi L 773

Ramsay M 17

Ramsebner R 427

Ramser J 217 (SR)

Randall S 150 (L)

Ravazzolo R 183

Regland B 113

Rehder H 334 (SR)

Reichardt S 790

Reichwald K 17

Reis A 17

Reuter K 188

Riccio A 95

Richards N 250

Ricksten A 113

Ridanpää M 439

Rimoin DL 439

Riou P 239

Ripa R 631, 887 (E)

Rivero JC 457

Robaszkiewicz M 100

Roberts RG 413

Robertson SP 865

Robledo M 457

Rockas S 439

Roizès G 833

Rojas CV 638

Romain DR 767

Romana S 699

Romein T 487 (SR)

Rommens JM 250

Romppanen E-L 569

Ronce N 516

Rosenthal A 17

Rossi A 516

Rötig A 289 (VP)

Rouleau GA 406, 773

Roux A-F 72

Rozet J-M 197

Ruault M 833

Ruiz-Llorente S 457

Russo S 516

Rustin P 289 (VP)

Ruszniewski P 100

Rymo L 113, 579
Sadeh M 226

Saffroy R 239

Sagie TL 226

Sahbatou M 320

Salem N 391 (SR)

Samuels ME 539

Sandig KR 790

Sandkuijl LA 271

Sankila E-M 339

Sans S 841

Santa Maria L 638

Santamaria G 183

Santos JL 638

Santos R 197

Satta D 320

Sauer C 449

Sayer AA 749

Sayı A 77 (SR)

Scanavini D 576

Schalling M 276

Scheurlen M 499

Schmid M 281 (SR)

Schmierer K 773

Schöfer C 427

Schollen E 643

Schreiber S 259

Schroer R 757

Schulte HD 741

Schultealbert A 753

Schürmann M 729

Schwartz CE 757

Schwartz K 157 (PG)

Schwartz M 197

Schwinger E 204, 729

Scotet V 100

Scriven PN 801

Seidel J 790

Senger G 790

Şengül B 786 (L)

Sermon K 591

Sevilla C 599

Sevon P 658

Sewry CA 413

Sham P 125, 857, 870

Shaw MA 767

Shen Y 484 (SR)

Shinawi M 145

Shizuka M 773

Shugart YY 133

Sibon I 773

Siest G 841

Simmons A 17

Simonelli F 197

Simpson S 167

Simpson SA 689 
Singer C 773

Sinnett D 62

Sistermans EA 487 (SR)

Sistonen P 439

Smith RJH 757

Sobol H 599

Société Française de Neurochirurgie 733

Solano AR 395 (SR)

Solassol j 462

Soler G 239

Soloway PD 6 (R)

Sørensen SA 213 (SR)

Sorrentino G 773

Soubrier F 553

Soubrier F 715

Sousa M 467

Southam L 562

Soytürk M 786 (L)

Spandidos DA 113, 579

Spentchian M 666

Starke H 790

Starostik P 499

Stegmann K 753

Stephani U 188

Sternberg D 851

Stevenson RE 757

Stöhr H 281 (SR)

Stoppa-Lyonnet D 599

Storch A 773

Strehl E 753

Stuart P 327

Stuhrmann M 729

Stumm M 790

Stuppia L 388 (SR)

Su C-C 495 (SR)

Su M-C 495 (SR)

Suarez-Merino B 857

Suefert J 499

Syddall HE 749

Szuhai K 427

Taillandier A 666

Tammur J 197

Tan Q 119
Tanaka M 773

Taskinen M-R 547

Taylor RW 141

Te Meerman GJ 197

Tejpar S 505

Telleria D 457

Tesei S 292

Testa F 197

Teugels E 879 (SR)

Thalheimer A 499

Thiery J-P 819

Thomas NS 707

Thomas-Anterion C 399

Thys S 883 (SR)

Tian Y 162

Tighe O 530

Timonen K 649

Tiret L 553, 715

Tomkins DJ 44

Topçu M 77 (SR)

Torrisi C 362

Törüner GA 77 (SR)

Touraine R 2 (PG)

Tournier-Lasserve E 733

Toutain A 516

Tranchemontagne J 516

Treis H 499

Trevisol-Bittencourt PC 773

Trijbels FJM 433

Troiano L 292

Trump D 516

Tsai C-C 495 (SR)

Tunca M 786 (L)

Turleau C 699

Turnbull DM 141

Ukraintseva SV 119

Ulaş Yavuzşen T 786 (L)

Underhill PA 521

Utermann G 351

Vainzof M 825

Vakkilainen J 537

Van Agtmael T 623

Van Bokhoven H 487 (SR)
Van Broeckhoven C 276

Van Camp G 197, 883 (SR)

Van Cutsem E 505

Vandenberghe A 297

van der Bruggen P 833

van der Kwast ThH 819

Van der Put NMJ 433

Vang M 381

Van Gestel S 276

Van Laer L 883 (SR)

Van Ommen G-JB 1 (E)

van Ommen G-JB 575

van Rhijn BWG 819

Van Steirteghem A 591

van Tilborg AAG 819

Vaupal JW 119

Vekemans M 699

Velissariou V 694

Verpillat P 399

Vervoort VS 757

Vicari E 362

Viggiani M 137

Viikari JSA 547

Vikkula M 375

Villard L 86 (SR), 223 (PG)

Visvikis S 553, 715, 841

Vitale E 95

Viville S 231

Volkmann J 773

Volleth M 790

von und zu Fraunberg M 649

Voorhees J 327

Vreugde S 339

Wachtler FJ 427

Wadelius C 197

Wallgren-Pettersson C 516

Walpole I 516

Walshe V 266

Wang AG 381

Wang Y-C 495 (SR)

Wang Y-P 484 (SR)

Watanabe M 773

Watanabe S 375
Weber BHF 197, 281 (SR), 449

Weil D 210, 391 (SR), 851

Weipoltshammer K 427

Weitzel J 395 (SR)

Werdelin LM 213 (SR)

Wilkie H 857

Williamson R 623

Wilson AF 133

Wiltshire S 177

Wimmer K 334 (SR)

Wissinger B 449, 865

Witte JS 82 (SR)

Wolter B 741

Wood NW 773

Wu X-R 484 (SR)

Wulff H 36

Wutz K 449

Xiong M 607

Yang $Y 52$

Yapijakis C 167

Yapijakis C 689

Yashin Al 119

Ylitalo K 547

Yntema HG 487 (SR)

Zahn R 217 (SR)

Zatz M 825

Zeniou M 2 (PG)

Zenner HP 95

Zertal-Zidani S 320

Zetterberg H 113, 579

Zeviani M 773

Zhang Y 266, 484 (SR)

Zhao H 162

Zimmer M 266

Zimmermann W 17

Zinger A 226

Zoeteweij MW 167, 689

Zou Y 162

Zrenner E 449

Zühlke C 204

Zwarthoff EC 819 